\section{A Simulink-based Channel Emulator for Underwater Acoustic Communications}

\section{Dey and N. Marchetti}

\begin{abstract}
The primary contribution of this paper is to develop a realistic yet mathematically tractable channel emulator for underwater acoustic communications (UWAC) channels. Our proposed emulator is based on the Wide-Sense Stationary Uncorrelated Scattering (WSSUS) assumption, where the channel samples are generated using finite sumof-sinusoids (SOS) with uniformly distributed phases. The MATLABbased Simulink platform and its associated block-set elements are used to generate numerical plots for amplitude distribution, Doppler frequency and channel impulse response. This Simulink-based design offers an added advantage over prevalent UWAC channel simulators, as it can be directly adapted to FPGA platform for hardware implementation.
\end{abstract}

Introduction: Underwater Acoustic Communication (UWAC) has recently gained a huge momentum due to its wide variety of applications including, monitoring and control of off-shore oil fields, oceanic environmental conditions or bio-geo-chemical processes etc. [1]. Despite favorable characteristics like low attenuation of sound waves, low signal absorption in underwater environments, UWAC channels pose significant challenges to effective communication [2].

There is no "typical" underwater communications environment as different geographical areas are affected by different physical parameters and processes. Therefore, system developers resort to extensive sitespecific measurement campaigns, trials, testing and validation of designed systems, which subsequently may fail to work in some other environment. Such procedures not only impose huge cost constraints, but also cannot ensure effectiveness in all environments. This establishes the need for a simple channel emulator for UWAC environments with as much realism as possible, significantly reducing design and performance prediction costs $[2,3]$.

While communicating over an UWAC channel, small differences in velocities of the sound waves lead to preferred propagation directions for the transmitted signal owing to the fluctuations in water temperature, salinity and density. A collection of transmission rays travels along each of the preferred directions and are referred to as path bundles. Such assortments of communication paths are reflected and delayed both at the base and the surface of the water body.

In the signal processing domain, the UWAC propagation channel demonstrates an impulse response comprising of multiple delayed reflections of the transmitted signal. Physical properties like amplitude, phase and position of these echoes change with time and frequencies of operation. These echoes can be grouped to form the channel impulse response (CIR). The time variations in CIR of an UWAC channel are very slow and are concentrated around frequencies below $10 \mathrm{~Hz}$. Additionally, Doppler shifts are introduced around fundamental frequencies through the movement of either the receive and/or transmit terminals. Therefore, variations occur both in the time and delay domains resulting in the socalled doubly-spread channel $[4,5]$.

The mathematical model of an UWAC channel needs to account for the motion-induced time-varying behavior and Doppler shifts in the acoustic propagation channel. A few channel emulators have been proposed in literature over the years $[6,7]$, the bulk of which uses physics based modeling $[8,9]$ while the rest uses replay-based methods [10]. The simulator in [8] uses a time dependent auto-regressive memory process to create the Doppler spectrum, which is approximated by the exponential distribution. This is not entirely realistic in spite of the visual similarity between the Fourier transform of the Doppler spectrum and exponential correlation function, since the auto-correlation function of the fading parameter of a linear time-variant (LTV) UWAC channel has no closedform expression. On the other hand, [10] uses scattering functions to drive the simulator. The scattering functions, in turn, are derived from extensive measurement campaigns only. In this case, the simulator also requires recognition and removal of time-varying Doppler prior to realistic channel emulation.

This paper proposes a channel emulator for UWAC channels, where channel samples are produced using the sum-of sinusoids (SOS) model. The channel model is based on the Wide-Sense Stationary Uncorrelated Scattering (WSSUS) assumption. From several measurement campaigns
$[8,11]$, it can be observed that the impulse response of an UWAC consists of a small number of uncorrelated discrete echoes. This enables us to use WSSUS model for complete channel characterization, where the echoes can be replaced by impulses. For realistic implementation, we assume that transmit and receive filters (root-Nyquist pairs) are attached before and after the channel respectively. The transmit-receive filter pair ensures elimination of any inter-symbol interference (ISI).

If we assume that the transmit and receive filters have bandwidths less or equal to the usable bandwidth of the corresponding communication path, uncorrelated echoes with infinite bandwidth can be accumulated to emulate the communication channel. Hence, the overall structure of our proposed emulator can be visualized as a discrete linear time-varying filter and here, we implement it using a tapped delay line model. We lodge the popular sum-of-sinusoids (SOS) model to produce the filter taps. The SOS model generates samples of the acoustic communication channel with Ricean distributed amplitude and stretched exponentially distributed Doppler power spectrum. The proposed emulator also accounts for Doppler shift due to drifting, vehicular and surface velocities and random time delays for each channel tap.

Channel Characterization: In order to model UWAC channels, LTV systems have been used extensively in literature due to processes such as tides, currents, moving transmitter / receiver / surface, and internal waves. Using LTV systems, the multipath channel over an UWAC link can be characterized in time domain using,

$$
h(\tau, t, f)=\sum_{p=1}^{P} h_{p} \gamma_{p}(f, t) \delta\left(\tau-\tau_{p}(t)\right)
$$

where $P$ is the total number of distinct echoes or path bundles encountered, with amplitudes $h_{p}$ and delays $\tau_{p}$. The fading parameter $\gamma_{p}(f, t)$ models the small-scale time variations of each path bundle. Time correlation function can be used to derive the statistical description of the random process $\gamma_{p}(f, t)$ if it is stationary in $t$. In that case, the channel Doppler spectrum can be modeled using the Fourier transform of the autocorrelation function of the fading parameter, $\gamma_{p}(f, t)$

$$
R_{p}(f, \Delta t)=\mathrm{E}\left[\gamma_{p}(f, t) \gamma_{p}^{*}(f, t+\Delta t)\right]
$$

where $*$ denotes the complex conjugate. The channel Doppler spectrum is approximated by a stretched exponential distribution,

$$
S_{p}(f, \eta)=\mathcal{F}\left(R_{p}(f, \Delta t)\right) \propto e^{-\left(\frac{|\eta|}{\lambda}\right)^{\zeta}}
$$

where $\mathcal{F}(\cdot)$ denotes Discrete Fourier Transform (DFT), $\eta$ are the Doppler frequencies, $\lambda$ is the parameter that matches the Doppler power spectrum to the exponential function, and $\zeta$ is the stretching exponent. There is no closed form expression for $R_{p}(f, \Delta t)$ and therefore, its Fourier transform, $\mathcal{F}\left(R_{p}(f, \Delta t)\right)$ is not exponentially distributed. Therefore, using time domain auto-regressive memory process to produce the stretched exponential spectrum, as done in [8], is neither accurate nor practicable. Hence, we resort to the SOS model for generating random processes directly from the Doppler spectrum.

Assuming stationarity and uncorrelated echoes with infinite bandwidth, the delay power spectrum of the channel and its frequency correlation function can be expressed as, $R_{h}(\tau)=\mathrm{E}\left[h(t, \tau) h^{*}\left(t, \tau^{\prime}\right)\right]=$ $\sum_{p=1}^{P} \mathrm{E}\left[\left|h_{p}(t)\right|^{2}\right] \delta\left(\tau-\tau_{p}\right) \quad$ and $\mathrm{E}\left[\mathcal{F}\left\{h\left(t, \tau^{\prime}\right)\right\} \mathcal{F}\left\{h^{*}(t, \tau)\right\}\right]=$ $\frac{1}{2 \pi} \sum_{p=1}^{P} \mathrm{E}\left[\left|h_{p}(t)\right|^{2}\right] e^{j 2 \pi\left(f-f^{\prime}\right) \tau_{p}}$ respectively. We note that the frequency correlation function depends only on the difference $f-f^{\prime}$. This means that wide-sense stationarity in the frequency domain is a direct consequence of the uncorrelated scattering assumption. As the individual path delays $\tau_{p}$ are uncorrelated, the time variations in a WSSUS channel can be calculated for each delay $\tau$ separately and the scattering function in the delay domain becomes,

$$
S(\tau, \eta)=\mathcal{F}\left\{\sum_{p=1}^{P} \mathrm{E}\left[h_{p}(t) h_{p}^{*}(t+\Delta t)\right] \delta\left(\tau-\tau_{p}\right)\right\}
$$

where the Fourier transform $\mathcal{F}\{\cdot\}$ is computed with respect to $\Delta t$. Correspondingly, $S\left(\tau_{p}, \eta\right)$ will represent the delay-domain scattering function of the $p$-th path with respect to its Doppler spectrum.

Discrete-Time Channel Model: A plethora of UWAC channel measurement campaigns like KAM, MACE, SPACE [8] conducted in different locations revealed that Ricean distribution offers precise 
characterization of the channel stochastic behavior. A variety of Ricean $K_{R}$ factors are encountered for different sets of reflected path bundles due to surface, bottom, surface-bottom, surface-bottom-surface combination etc. Therefore, in this section, we will be using the SOS model to generate channel samples whose amplitudes follow Ricean distribution. The sumof-sinusoids (SOS) model can be used to emulate a WSSUS channel with a specific scattering function, where the channel samples $h_{p}[i]=h_{p}\left(i T_{s}\right)$ are generated according to,

$h[i]=\frac{1}{\sqrt{M\left(1+K_{R}\right)}}\left[\sum_{m=1}^{M} e^{j\left(\phi_{m}+2 \pi f_{D} i T_{s}\right)}+\sqrt{K_{R}} e^{j\left(\phi_{0}+2 \pi f_{0} i T_{s}\right)}\right]$

where $T_{s}$ is the sample time interval used to represent the continuous time variable $t, M$ is the number of multipaths to model each channel tap, $\phi_{m}$ are random phases uniformly distributed in the interval $[0,2 \pi]$ with initial phase $\phi_{0}$ and $f_{D}$ are the $M$ Doppler frequencies contributing to each channel tap [12]. In (5), $K_{R}$ is the Ricean factor given by the ratio of the power in the direct line-of-sight (LOS) path to that in other scattered paths and $f_{0}$ is the Doppler frequency of the LOS path in each channel tap $^{1}$.

In order to generate (5), $M$ Doppler frequencies $f_{D}$ are needed to be selected from the Jakes spectrum such that they are the Fourier transforms of (3). The probability density function (PDF) of the values of $f_{D}$ can be subsequently given by,

$$
S(\eta)=\frac{1}{2 \alpha} e^{-(|\eta| / \lambda)^{\zeta}} .
$$

Equation (6) can be used to draw the values of $f_{D}$ using the inverse transform sampling lemma which states that, if input $X$ has a distribution function of $f(x)$, the output will have a uniformly distributed cumulative distribution function $(C D F) F(X)$ within the limits $[0,1]$. Therefore we can generate samples following $f(x)$ by choosing $y$ uniform in $[0,1]$, and applying $x=F^{-1}(y)$. Integrating (6), we find the CDF of the Doppler frequencies as,

$$
F\left(f_{D}\right)=\int_{-\infty}^{f_{D}} \frac{1}{2 \lambda} e^{-(|\eta| / \lambda)^{\zeta}} \mathrm{d} \eta=\frac{1}{2 \beta} \Gamma\left[\frac{1}{\zeta},-\left(-\frac{f_{D}}{\lambda}\right)^{\zeta}\right]
$$

where $\Gamma(\cdot, \cdot)$ is the upper incomplete Gamma function.

An arbitrary probability distribution can be extracted from a uniform distribution using inverse transform sampling. Using $F\left(f_{D}\right)=u$ as a uniform distribution in $[0,1]$, the transformation,

$$
f_{D}=-\lambda\left[\sum_{k=0}^{1 / \zeta-1}(k+1) ! W\left\{\frac{1}{k}\left(\frac{(\bmod (2 u, 1)) \zeta^{2}}{\zeta-1}\right)^{1 / k}\right\}\right]^{1 / \zeta}
$$

produces stretched exponential distributed Doppler frequencies, where 'mod' refers to the modulo operation which returns the remainder after division of $2 u$ by $1, W$ denotes the Product-Log or Lambert-W function. In order to implement a discrete frequency-selective channel model, the channel impulse response can be expressed as,

$$
h(t ; \tau)=\sum_{p=1}^{P} c_{p}(t) \delta\left(\tau-\tau_{p}\right)
$$

where $c_{p}(t)$ are the fading coefficients. However, the model in (9) is incomplete, as it has infinite bandwidth (revealed by use of delta function). So to use it for system implementation, transmit and receive filtering are to be considered.

Let us consider a communication system where $x_{i}$ complex transmit samples travel through a pulse shaping transmit filter with impulse response $g(t)$, the propagation channel, and a matched receive filter. If multiples of the symbol time $T$ or another sampling time $T_{s}$ are used for sampling the output signal $z(t)$, the overall communication system becomes a discrete linear time-varying filter. Such a system can be characterized using a tapped delay line model, where $l=1,2, \ldots L$ are the indices of the filter taps considered for the tapped delay line model. In order to accomplish that, we need to generate the values of the filter taps, $f_{l}\left(k T_{s}\right)$ which can be achieved using digital filtering with a sampling

${ }^{1}$ The channel statistics formulated in (5) becomes equivalent to those of (4) for $M \rightarrow \infty$. However, in practicality, stationarity can be achieved even for $M<\infty$. Consequently, a good approximation can be achieved for $M>100$. rate sufficient to model the usable channel bandwidth after synthesizing the channel model of (9).

The filter taps with initial delay of $\tau_{0}$ and average power levels of $\left\{P_{p}\right\}$ in $P$ path bundles can be generated in a way similar to the time-varying path weights of (5) as,

$$
\begin{aligned}
& f_{l}[k]=\sum_{p=1}^{P} \sqrt{\frac{P_{p}}{M\left(1+K_{R}\right)}}\left[\sum_{m=1}^{M} e^{j\left(\phi_{m, p}+2 \pi f_{m, p}\left(k T_{s}-\tau_{p}\right)\right)}\right. \\
& \left.\times g\left(l T_{s}-\tau_{p}\right)+\sqrt{K_{R}} e^{j\left(\phi_{0, p}+2 \pi f_{0, p}\left(k T_{s}-\tau_{0}\right)\right)} g\left(l T_{s}-\tau_{0}\right)\right] .
\end{aligned}
$$

Moreover, if the drift velocity between two terminals is given by $v$, any time period $t$ will appear to have extended to a time period equal to $t+$ $v t / c=(1+a) t$, where $a$ is referred to as the Mach number and $c \sim 1500$ $\mathrm{m} / \mathrm{s}$ is the speed of sound in water. In that case, (10) can be modified to accommodate for the Doppler shift,

$$
\begin{aligned}
f_{l}[k]=\sum_{p=1}^{P} & \sqrt{\frac{P_{p}}{M\left(1+K_{R}\right)}}\left[\sum_{m=1}^{M} e^{j\left(\phi_{m, p}+2 \pi f_{m, p}\left(a k T_{s}-\tau_{p}\right)\right)}\right. \\
& \left.\times g\left((k-l+a k) T_{s}-\tau_{p}\right)\right]+e^{j\left(\phi_{0, p}+2 \pi f_{0, p}\left(a k T_{s}-\tau_{0}\right)\right)} \\
& \left.\times \sqrt{K_{R}} g\left((k-l+a k) T_{s}-\tau_{0}\right)\right] .
\end{aligned}
$$

It is to be noted here that the choice of $M$ should be a trade-off between stationarity of the generation model in (11) and the computational complexity of the emulator.

Analysis of UWAC channel impulse responses gathered during sea trials conducted in numerous measurement campaigns has shown that underwater communication channels can hardly ever be modeled as a WSSUS stochastic process. However, even in case of a non-WSSUS channel, it is possible to determine the period of time ('Stationary Time') and frequency ('Stationary Bandwidth') range, in which the WSSUS assumption is locally satisfied [13]. If we define $R_{h}(t, \Delta \tau)$ as the autocorrelation function of $h(t, \tau)$ in time domain, then Stationary Time can be defined as the maximum observation time interval $T_{D}$, for which $R_{h}(t, \Delta \tau)$ is stationary and the mean value of $h(t, \tau)$ is constant. Consequently, if we define $R_{p}(f, \Delta t)$ as the autocorrelation function of $\gamma_{p}(f, \Delta t)$ (refer to (1) and (2)), then Stationary Bandwidth can be defined as the maximum frequency range $B_{D}$, in which $R_{p}(f, \Delta t)$ is stationary and mean value of $\gamma_{p}(f, \Delta t)$ is constant.

Channel Emulator: The SOS channel representation in (11) can be modeled using Simulink. The Simulink model for a Ricean (or Rayleigh) distributed channel with impulse response of up to 15 channel taps at discrete time delays is demonstrated in Fig. 1 (the taps are assumed to be non-overlapping). Separate Matlab functions have been used to generate Doppler shifts and Doppler frequencies. Matlab functions are also included in the Simulink model in Fig. 1 to characterize the time-dependent path gains $c_{p}(t)$ in (9) with programmable tap delays $\tau_{p}$.

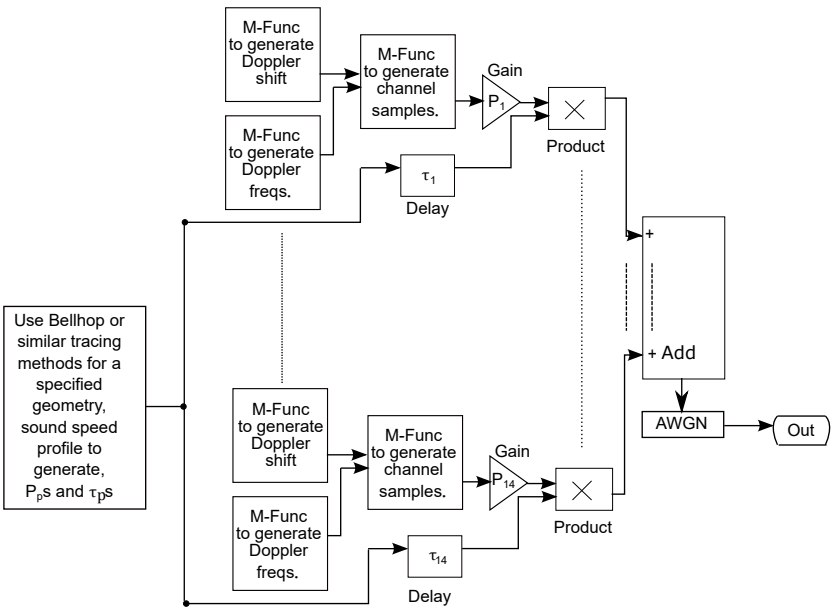

Fig. 1. Simulink-based SOS channel simulation model. 
Fig. 2 shows sample trajectories generated over a 3-second interval with the Simulink model and $M=1000$, together with the sample amplitude and Doppler frequency distributions using parameters tabulated in Table 1. These parameters are adopted from the ones used for distribution fitting to the measurement campaigns conducted in $[8,10]$.

Table 1: Parameter Set for Emulation

\begin{tabular}{c|c|c}
\hline \hline$K_{R}$ & $\lambda(\mathrm{Hz})$ & $\zeta$ \\
\hline \hline 3 & $0.024,0.44$ & $0.39,0.63$ \\
\hline 5 & $1.27,3.08$ & $0.50,1.16$ \\
\hline \hline
\end{tabular}
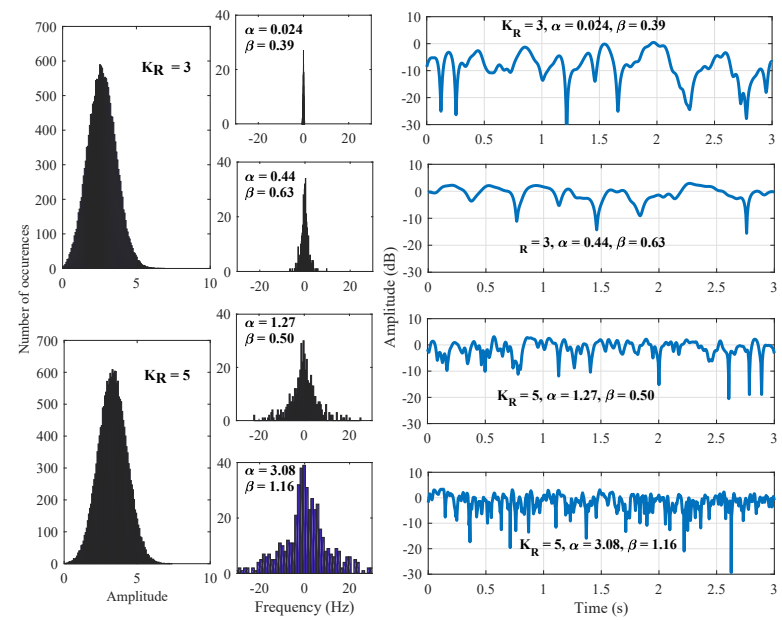

Fig. 2 Sample trajectory of the channel emulator with sets of emulated multipath gain over fixed duration of 3-s shown on the right side, histograms of path amplitudes and Doppler frequencies on the left side.

The proposed channel emulator is developed using the Simulink platform and is created using the Simulink block-set elements. This emulator can be used to run simulations in Simulink which can also be converted to RTL code, IP catalogue items, or even bit-streams for JTAG co-simulations. This provides an added advantage over prevalent UWAC channel simulators [8-10] as this Simulink-based emulator can be directly ported to the FPGA platform for hardware implementation. Our emulator can input data symbols, filter, upsample and upconvert and finally downconvert and downsample them after being received over an emulated UWAC link. An example plot for channel impulse response generated using the proposed channel emulator is presented in Fig. 3.
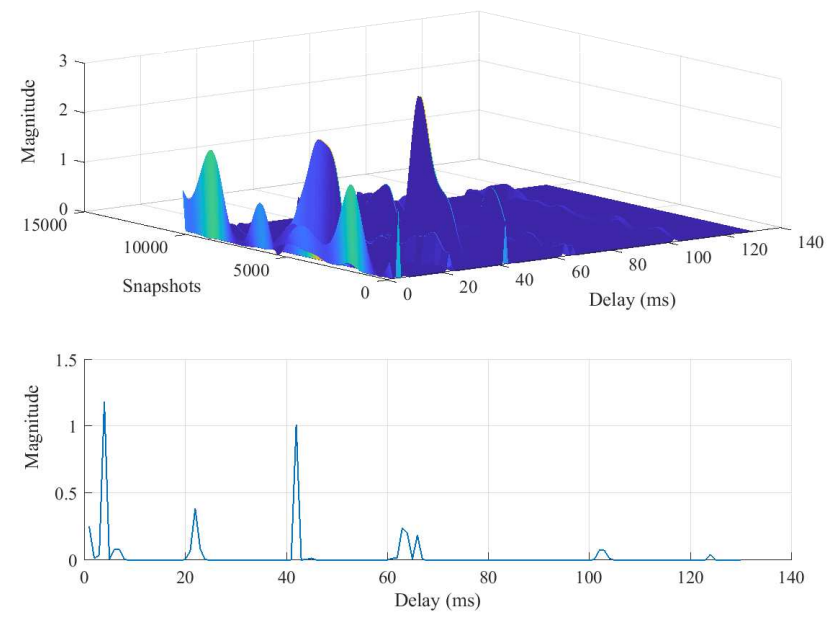

Fig. 3 Generated CIR with the top figure showing response for 10 channel taps and the bottom one presenting the evolution of a single channel tap.

Conclusion: In this paper, a novel SOS-based channel model is proposed for UWAC system to describe the WSSUS channel characteristics. Based on the proposed model, a channel emulator is developed using the Simulink platform and block-sets which can be used to generate practical UWAC links. The proposed emulator accounts for both time and delay domain variations in the channel and consequently, will be able to offer realistic prediction of performances of different channel estimation, precoding and detection algorithms. The Simulink model developed can be easily extended to other platforms, coding techniques and co-simulations for tractable hardware implementation. If needed, details on other physical processes like pathloss, shadowing, surface motion, absorption etc. can also be included to this proposed emulator just by adding Simulink blocksets.

Acknowledgment: This work is supported by the EDGE grant 13236/203377 under Marie Skodowska-Curie COFUND Actions.

I. Dey and N. Marchetti (CONNECT, Trinity College Dublin, Ireland)

E-mail: deyi@tcd.ie

\section{References}

1 D. Kilfoyle, and A. B. Baggeroer, "The State of the Art in Underwater Acoustic Telemetry," IEEE J. Oceanic Engineering, vol. 25, no. 1, pp. 427, 2000.

2 J. Preisig, and G. Deane, "Surface wave focusing and acoustic communications in the surf zone," J. Acoust. Soc. Am., vol. 116, no. 4, pp. 2067-2080, 2004

3 R. A. van Walree, T. Jenserud, and R. Otnes, "Streched-exponential Doppler spectra in underwater acoustic communication channels," J. Acoust. Soc. Am., vol. 128, no. 5, Nov. 2010

4 C. S. Clay, and H. Medwin, Acoustical Oceanography: Principles and Applications, John Wiley \& Sons, New York, NY, 1977.

5 F. Jensen, W. Kuperman, M. Porter, and H. Schmidt, Computational Ocean Acoustics, Springer, New York, NY, 2000.

6 C. Petrioli, R. Petroccia, J. Potter, and D. Spaccini, "The SUNSET Framework for Simulation, Emulation and at-sea Testing of Underwater Wireless Sensor Networks," Journal Ad Hoc Networks, 2015, vol. 34, issue C, pp. 224-238.

7 Y. Zhu, S. Le, L. Pu, X. Lu, Z. Peng, J.-H. Cui, and M. Zuba, "AquaNet Mate: A real-time virtual channel/modem simulator for Aqua-Net," in Proc. MTS/IEEE Ocean, Bergen, Norway, Jun. 2013.

8 P. Qarabaqi, and M. Stojanovic, "Statistical Characterization and Computationally Efficient Modeling of a Class of Underwater Acoustic Communication Channels," IEEE J. of Oceanic Engg., vol. 38, no. 4, pp. 701-717, Oct. 2013.

9 M. Chitre, "A high-frequency warm shallow water acoustic communications channel model and measurements," J. Acoust. Soc. Amer., vol. 122, no. 5, pp. 2580-2586, Nov. 2007.

10 P. A. van Walree, T. Jenserud, and M. Smedsrud, "A discrete-time channel simulator driven by measured scattering functions," IEEE J. Sel. Areas Commun., vol. 26, no. 9, pp. 1628-1637, Dec. 2008.

11 J. Zhang, J. Cross, and Y. R. Zheng, "Statistical channel modeling of wireless shallow water acoustic communications from experimental data," in Proc. Military Commun. Conf., 2010, pp. 2412-2416.

12 N. Eskandari, M. Bashir, D. Truhachev, C. Schlegel and J.-F. Bousquet, "Improving the quality of underwater acoustic channel via beamforming", in Proc. 2018 OCEANS - MTS/IEEE, Kobe Techno-Oceans (OTO), 2018.

13 I. Kochanska, and I. Nissen, "Limitations of WSSUS modeling of stationary underwater acoustic communication channel," Annual Journal of Hydroacoustics, Gdansk 2016, vol. 19, pp. 229-238. 